\title{
Post-traumatic osteomyelitis of the clavicle: A case report and review of literature
}

\author{
Chenicheri Balakrishnan MD, Christopher Vashi MD, Ollie Jackson MD, Jason Hess MD
}

C Balakrishnan, C Vashi, O Jackson, J Hess. Post-traumatic osteomyelitis of the clavicle: A case report and review of literature. Can J Plast Surg 2008;16(2):89-91.

\begin{abstract}
Osteomyelitis of the clavicle is a rare form of infection occurring from hematogenous spread or trauma. This has been reported following head and neck surgery, and subclavian catheter placement. In traumatic cases, the management involves removal of bone fixation, debridement of the bone and coverage with a muscle flap.
\end{abstract}

Key Words: Clavicle; Osteomyelitis

\section{Une ostéomyélite post-traumatique de la clavicule : Rapport de cas et analyse bibliographique}

L'ostéomyélite de la clavicule est une rare forme d'infection découlant d'une propagation hématogène ou d'un traumatisme. On en a déclaré des cas après une opération de la tête et du cou et l'installation d'un cathéter sous-clavier. Dans les cas de traumatisme, la prise en charge inclut l'extraction de la fixation osseuse, le débridement de l'os et le recouvrement avec un lambeau musculaire.

$\mathrm{O}$

steomyelitis of the clavicle is a rare condition that is difficult to diagnose. Despite modern surgical techniques and advanced antimicrobial therapy, osteomyelitis remains a difficult and challenging problem. Chronic osteomyelitis constitutes the result of the unfavourable evolution of the acute form. It generally involves the long bones and is often characterized by an osseous sequestration at the origin of cutaneous fistulas, and pathological fractures. Surgical debridement, muscle flaps and long-term use of antibiotics are associated with good clinical outcome.

\section{CASE PRESENTATION}

A 24-year-old man presented to the orthopedic service with a draining sinus over the left clavicle with an exposed plate. His past surgical history revealed an open fracture of the clavicle following a fall three years previously. At that time, he underwent open reduction and internal fixation of the fracture. His internal fixation plate was removed (Figure 1) and the patient was started on antibiotics empirically. Cultures from the screw holes grew Staphylococcus aureus. A decision was made to cover the bone with a muscle flap and to treat him with intravenous antibiotics for six weeks. He underwent closure of exposed bone with a pectoralis major muscle flap (Figures 2 and 3) and the skin was subsequently closed over the muscle flap (Figure 4).

\section{DISCUSSION}

Pain and swelling of the medial end of the clavicle may be associated with radiographic sclerosis. The most common causes are osteoarthritis, infection and condensing osteitis of the clavicle. Distinguishing between these entities can be difficult clinically and radiologically. Infection of the bone locus creates an increase in the intramedullary pressure due to the build up of

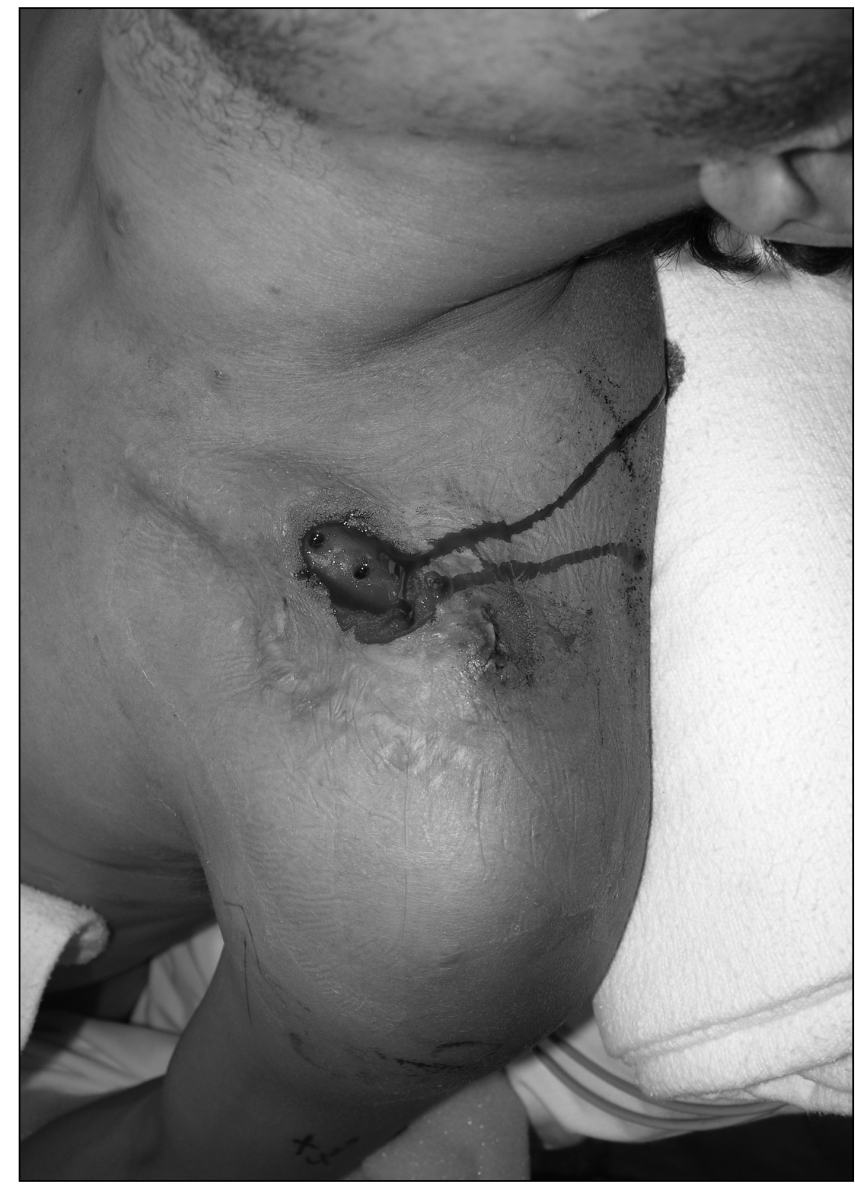

Figure 1) Exposed bone after removal of plate and screws 


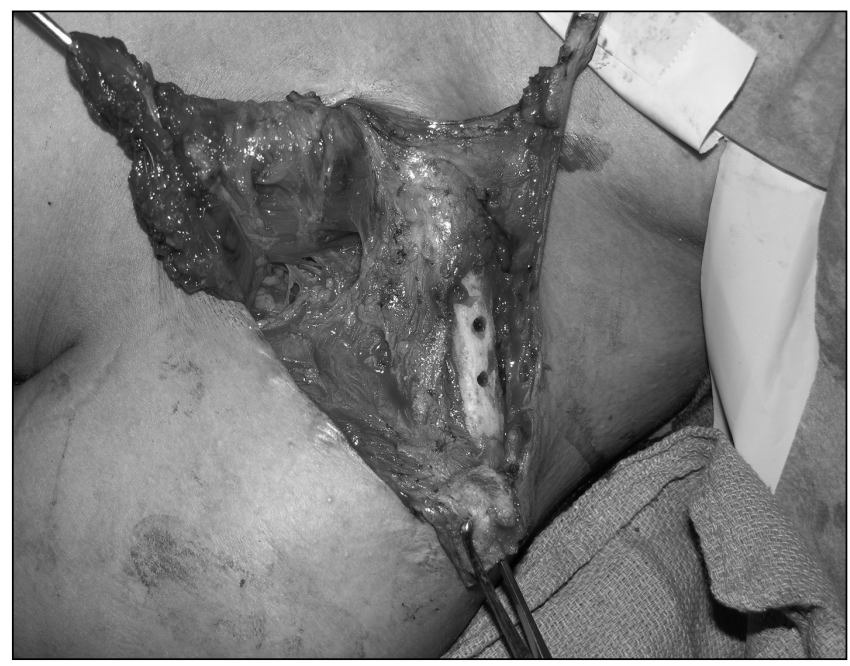

Figure 2) Pectoralis muscle elevated

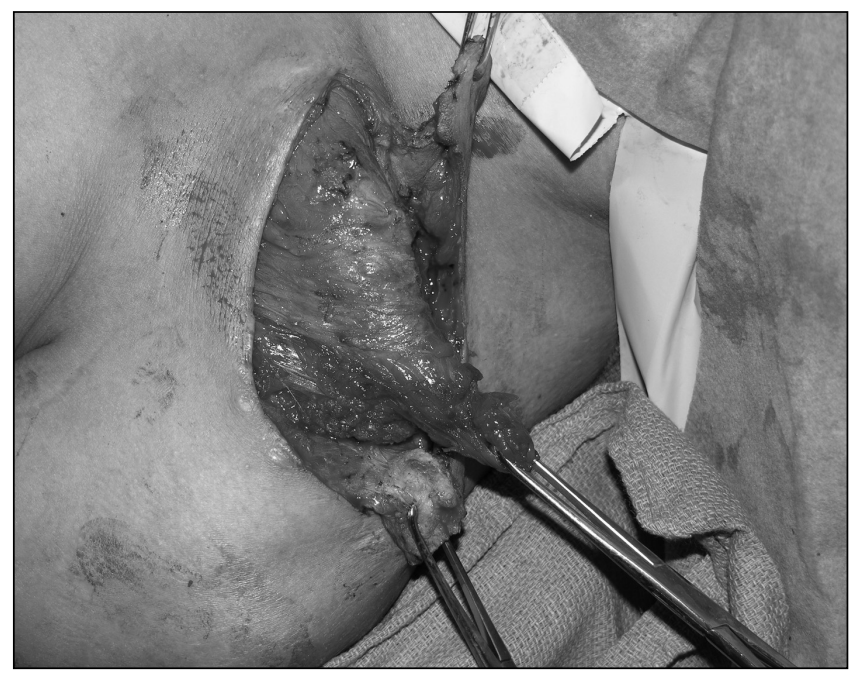

Figure 3) Clavicle covered with the muscle

inflammatory exudates that eventually strips the periosteum, leading to vascular thrombosis followed by bone necrosis and the formation of sequestra. Necrosis of the large segments of bone usually leads to formation of sequestrum. These sequestra, which contain infected material, are surrounded by sclerotic bone that is relatively avascular. The Haversian canals are blocked by scar tissue, and the bone itself is surrounded by thickened periosteum and scarred muscle. Antibiotics cannot penetrate these relatively avascular tissues and are hence ineffective in clearing the infection. New bone formation, known as the involucrum, occurs at the same time. Multiple openings appear in this involucrum, through which exudates and debris from the sequestrum pass via the sinuses. A periosteal reaction acts to circumscribe the sequestrum.

Chronic osteomyelitis is a severe, persistent, and sometimes incapacitating infection of bone and bone marrow. It is often a recurring condition because it is difficult to treat definitively. This disease may result from inadequately treated acute osteomyelitis, a hematogenous spread, trauma, iatrogenic causes such as joint replacements and the internal fixation of fractures, compound fractures, infection with organisms such as

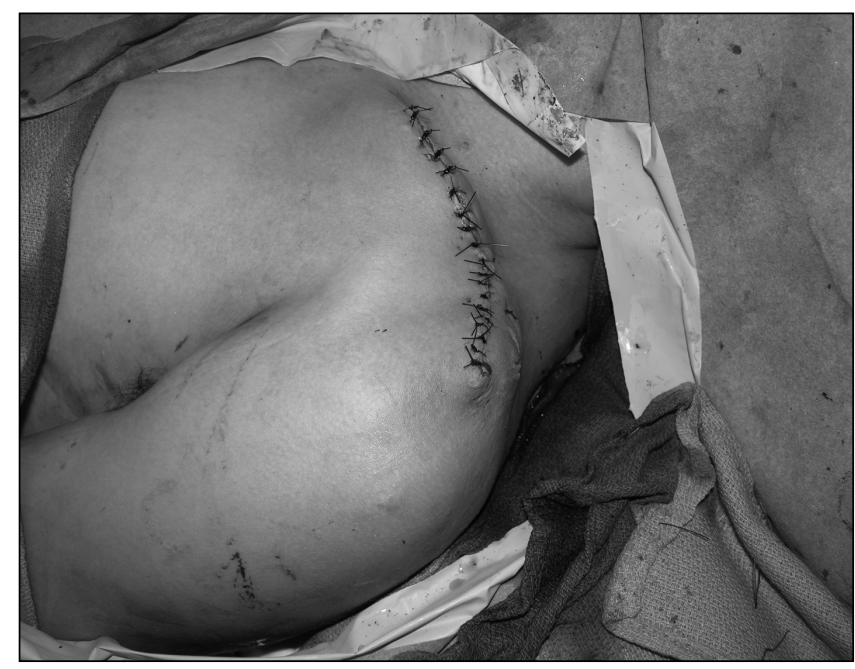

Figure 4) Closure of wound

Mycobacterium tuberculosis and Treponema species (syphilis), and contiguous spread from soft tissues, as in diabetic ulcers or ulcers associated with peripheral vascular disease. Osteomyelitis is a process of devascularization of the periosteal and endosteal arterial system secondary to the two mechanisms of the infection: the thrombophlebitis and the bone infection itself. In the absence of an early diagnosis and an adapted treatment, the result of this process is necrosis of the bone. These necroses can evolve to the resorption of the mortified zone or its detachment with constitution of sequestrations. The passage to chronic osteomyelitis is the result of the unfavourable evolution of the acute form. Nevertheless, certain authors $(1,2)$ described chronic forms from the start. Chronic osteomyelitis can lead to amyloidosis $(1,2)$.

Osteomyelitis of the clavicle is a rare entity and can occur as a complication of head and neck surgery (3-5), and of subclavian vein catheterization (6-8). Soft tissue infections around the puncture site occur frequently and respond well to topical and antibiotic treatment. If symptoms persist for several weeks, osteomyelitis or sternoclavicular pyoarthrosis should be considered. Making the diagnosis of osteomyelitis can be difficult. No one diagnostic test can definitively rule in or sufficiently rule out osteomyelitis. Acute osteomyelitis from hematogenous spread has a biphasic occurrence pattern, peaking in those patients younger than 17 years of age and older than 50 years of age. Certain organisms, $S$ aureus among them, have virulence factors that make them particularly well adapted to infecting bone. In vitro studies have suggested that $S$ aureus can live intracellularly within osteoclasts. In addition, $S$ aureus has the ability to upregulate the production of certain adhesion molecules, which facilitate its attachment to cartilage, especially in the early stages of infection. The production of local cytokines can impair the host's immune response, making osteomyelitis a difficult infection to treat (9).

Pain and swelling of the clavicle may be associated with radiographic sclerosis. The most common causes are osteoarthritis, infection and condensing osteitis of the clavicle. Laboratory results that can be useful in the diagnosis of osteomyelitis include an elevated white blood cell count and an elevated erythrocyte sedimentation rate. Blood cultures are positive in approximately $50 \%$ of cases of acute osteomyelitis, 
and the presence of radiographic evidence coupled with positive tissue cultures usually confirms the diagnosis. Distinguishing between these clinically and radiologically can be difficult, but computed tomography (CT) and magnetic resonance imaging are often useful for their differentiation. Radiological findings should be correlated with clinical features, which together should enable a confident diagnosis to be made. Plain radiographic findings in acute or subacute osteomyelitis include deep soft tissue swelling, a periosteal reaction, cortical irregularity and demineralization. The chronic phase of the disease is characterized by thick, irregular, sclerotic bone interspersed with radiolucencies, an elevated periosteum and chronic draining sinuses.

CT images sometimes reveal soft-tissue edema and/or bone destruction not seen on plain images, particularly in the setting of acute osteomyelitis. Bone sclerosis, demineralization and periosteal reactions are usually well-depicted in chronic osteomyelitis. CT scans also help in evaluating the need for surgery as well as providing vital information about the extent of disease. CT is also an important modality in image-guided biopsy.

Magnetic resonance imaging findings in osteomyelitis are usually due to the replacement of marrow fat by water secondary to edema, exudate, hyperemia and bone ischemia. Findings include the following: decreased signal intensity in the involved bone on T1-weighted images, increased signal intensity in the involved bone on T2-weighted image, and increased signal intensity in the involved bone on short-tau inversion recovery (STIR) images. Granulation tissue is hypointense on T1-weighted images and hyperintense on T2-weighted and STIR images. It also shows gadolinium enhancement. Similarly, draining sinuses and soft-tissue inflammation are hypointense on T1-weighted images and hyperintense on T2weighted and STIR images; however, they do not show gadolinium enhancement $(10,11)$.

Despite modern surgical techniques and advanced antimicrobial therapy, osteomyelitis remains a difficult and challenging problem. Long-term intravenous antibiotic therapy (six to eight weeks) has been used to successfully treat cases of hematogenously spread osteomyelitis (2). With appropriate therapy, only approximately $5 \%$ of hematogenous cases will progress to chronic osteomyelitis. Prompt therapy can avoid the dreaded complication of necrosis of the involved bone. Surviving bone adjacent to and involving the prior site of osteomyelitis is frequently osteoporotic as a result of the infection. In most patients, the return to normal activity after resolution of the infection will enable the bone to regain its initial density. In time, adequately treated osteomyelitis can be indistinguishable from adjacent, normal, bone. Wide surgical debridement was the mainstay of treatment in the chronic conditions, with antibiotic therapy playing a secondary role. Myocutaneous flaps may be required after surgical debridement of the necrotic bone for wound closure. This also enables antibiotic penetration to the bone. In chronic osteomylitis secondary to open fractures, patients will not respond to antibiotics unless the fixation device is removed. This is due to the organism sticking to the biomaterials and is covered by a glycocalyx biofilm that presents a barrier to the antibiotics (9).

The present patient underwent removal of the hardware, debridment of bone to bleeding bone and coverage with a pectoralis major muscle flap with closure of the skin along with a long-term course of intravenous antibiotics. He has remained symptom free at 12 months.

\section{REFERENCES}

1. Cierny G 3rd, Mader JT, Penninck JJ. A clinical staging system for adult osteomyelitis. Clin Orthop Relat Res 2003;414:7-24.

2. Cierny G, Mader JT. Adult chronic osteomyelitis. Orthopedics 1984;7:1554-64.

3. Alessi DM, Sercarz JA, Calceterra TC. Osteomyelitis of the clavicle. Arch Otolaryngol Head Neck Surg 1988;11:1000-2.

4. Granick MS, Ramasastry SS, Goodman MA, Hardesty R. Chronic osteomyelitis of the clavicle. Plast Reconstr Surg 1989;84:80-4.

5. Winslow CP, Meyers AD. Clavicular osteomyelitis as a complication of head and neck surgery Ann Otol Rhinol Laryngol 1998;107:720-5.

6. Lee YH, Kerstein MD. Osteomyelitis and septic arthritis. A complication of subclavian vein catheterization N Engl J Med 1971;285:1179-80.

7. Klein B, Mittelman M, Katz R, Djaldetti M. Osteomyelitis of both clavicles as a complication of subclavian venipuncture. Chest 1983;83:143-4.

8. Watenkumakom C. Serratia marcescens osteomylitis of the clavicle and sternocclavicular arthritis complicating infected indwelling subclavian vein catheter. Am J Med 1986;80:753-4.

9. Crémieux AC, Carbon C. Experimental models of bone and prosthetic joint infections. Clin Infect Dis 1997;25:1295-302.

10. Alazraki NP. Radionucletide imaging in the evaluation of infection and inflammatory disease. Radiol Clin North Am 1993;31:783-94.

11. Gerscovich EO, Greenspan A. Osteomyelitis of the clavicle: Clinical, radiologic, and bacteriologic findings in ten patients. Skeletal Radiol 1994;23205-10. 